\title{
NÚMERO DE CASOS CONFIRMADOS DE TUBERCULOSE NO BRASIL ENTRE 2007 E 2016
}

\section{ARTIGO ORIGINAL}

SILVA, Fábio Tenório ${ }^{1}$

FECURY, Amanda Alves ${ }^{2}$

UTZIG, Ingrid Lara de Araújo ${ }^{3}$

OLIVEIRA, Euzébio ${ }^{4}$

DENDASCK, Carla Viana ${ }^{5}$

DIAS, Claudio Alberto Gellis de Mattos ${ }^{6}$

SILVA, Fábio Tenório. Et al. Número de casos confirmados de tuberculose no Brasil entre 2007 e 2016. Revista Científica Multidisciplinar Núcleo do Conhecimento.

1 Técnico em mineração pelo Instituto de Ensino Básico, Técnico e tecnológico do Amapá (IFAP).

${ }^{2}$ Biomédica, Doutora em Doenças Topicais, Professora e pesquisadora do Curso de Medicina do Campus Macapá, Universidade Federal do Amapá (UNIFAP).

3 Licenciada em Letras/Inglês, Especialista em Língua Inglesa, Professora e pesquisadora do Instituto de Ensino Básico, Técnico e tecnológico do Amapá (IFAP). ${ }^{4}$ Biólogo, Doutor em Doenças Topicais, Professor e pesquisador do Curso de Educação Física da, Universidade Federal do Pará (UFPA).

5 Teóloga, Doutora em Psicanálise, pesquisadora do Centro de Pesquisa e Estudos Avançados- CEPA.

${ }^{6}$ Biólogo, Doutor em Teoria e Pesquisa do Comportamento, Professor e pesquisador do Curso de Licenciatura em Química do Instituto de Ensino Básico, Técnico e tecnológico do Amapá (IFAP). 
Ano 05, Ed. 04, Vol. 04, pp. 94-104. Abril de 2020. ISSN: 2448-0959, Link de acesso: https://www.nucleodoconhecimento.com.br/saude/casos-confirmados-detuberculose, DOI: 10.32749/nucleodoconhecimento.com.br/saude/casosconfirmados-de-tuberculose

\section{RESUMO}

A tuberculose é uma doença infecciosa causada pela bactéria Mycobacterium tuberculosis. Ela está entre as doenças que mais matam no mundo. Segundo a Organização Mundial de Saúde (OMS), a Ásia e a África são os continentes que apresentam o maior número de casos confirmados do planeta. O objetivo deste trabalho foi mostrar o número de casos confirmados de tuberculose no Brasil entre 2007 e 2016. A pesquisa foi realizada no banco de dados DATASUS (http://datasus.saude.gov.br/). Os resultados mostram que o número de casos confirmados da doença se manteve estável por nove anos (2007 a 2015), apresentando uma queda acentuada no último ano pesquisado; a região sudeste possuiu o maior número de casos confirmados, seguido do nordeste do país, sul, norte e centro-oeste, respectivamente; as pessoas na faixa etária entre 20 e 59 anos de idade apresentam o maior número de casos; o gênero masculino é aproximadamente duas vezes mais acometido de tuberculose que o feminino; e a tuberculose pulmonar possuiu números muito superiores que a forma extrapulmonar e os casos de pessoas com os dois tipos da doença. A aglomeração de pessoas facilita a propagação da bactéria $M$. tuberculosis. O número de casos da tuberculose pulmonar foi maior que a quantidade de casos do tipo extrapulmonar porque ela é transmitida pelo ar de forma rápida em ambientes com muitas pessoas. Indivíduos adultos parecem ficar mais suscetíveis à tuberculose, pois não existe campanha de vacinação para esta faixa etária. Entretanto as chances de cura são altas quando se utiliza o tratamento correto. Parece ter ocorrido uma melhoria na organização da saúde do Brasil e um melhor preparo dos profissionais da área da saúde no atendimento da tuberculose.

Palavras-chave: Tuberculose, classificação, epidemiologia. 


\section{INTRODUÇÃO}

A tuberculose é uma doença infecciosa causada pela bactéria Mycobacterium tuberculosis. Ela está entre as doenças que mais matam no mundo, com 122 casos por 100000 habitantes em 2012. Segundo a Organização Mundial de Saúde (OMS), a Ásia e a África são os continentes que apresentam o maior número de casos confirmados do planeta (Mjid et al., 2015).

O tabagismo e algumas infecções como o HIV, por exemplo, aumentam as chances do desenvolvimento da tuberculose e, mesmo não sendo uma doença hereditária, o ambiente familiar é um grande facilitador da transmissão da bactéria. Assim, muitos cuidados devem ser tomados em casas onde alguém está infectado (Mjid et al., 2015).

A tuberculose é transmitida pelo contato pelo ar entre pessoas. $O$ risco de adquirir a doença é definido pelo grau de aproximação de pessoas infectadas. Apesar de inúmeras estratégias adotadas pela OMS para erradicá-la, inúmeros fatores como a imigração e o individualismo populacional, por exemplo, atrapalham. Em ambientes coletivos, é necessário isolar as pessoas infectadas e o cuidado deve ser maior em hospitais (Abouda et al., 2014).

Existem dois tipos de tuberculose: pulmonar (TB) e extrapulmonar (TBE), podendo haver casos de pessoas com os dois tipos. A diferença entre elas consiste na localização do seu desenvolvimento. A TB tem sua concentração e desenvolvimento nos pulmões. A TBE tem o desenvolvimento da bactéria em outras partes do corpo (Daronco et al., 2012; Mjid et al., 2015).

Os sintomas da TB e da TBE podem ser associados ou individuais. Pessoas contaminadas com TB podem apresentar tosse por mais de três semanas, suor noturno, febre, falta de apetite, dores no peito e perda de peso. Os sintomas da TBE dependem do local onde o $M$. Tuberculosis se instala. Quando a bactéria habita a pleura pulmonar, por exemplo, além dos sintomas apresentados na tuberculose pulmonar, são acrescidos falta de ar e dor torácica (Daronco et al., 2012). 
O diagnóstico da TBE é mais complicado que o da TB devido ao menor número de bactérias que este quadro apresenta. Os métodos desenvolvidos para diagnosticar a doença são radiografia do tórax para identificar lesões no pulmão; teste sanguíneo para ver se os glóbulos brancos estão produzindo maiores quantidades de proteínas que atrapalham a replicação de bactérias; e o teste Xpert MTB/RIF, um método moderno que se baseia na avaliação da presença da bactéria a partir da coleta e incubação do escarro do paciente (Abouda et al., 2014; Daronco et al., 2012).

O tratamento da tuberculose ocorre a partir de medicamentos que atacam a Mycobacterium tuberculosis. Ele deve ser cotidiano, feito em jejum e pela manhã. A OMS declarou efetivos quatro possíveis remédios para o tratamento da doença na fase inicial: isoniazide, rifampicine, pyrazinamide e ethambutol. A primeira parte do tratamento dura geralmente quatro meses e deve ser acompanhada pelo médico responsável para evitar complicações (Dhahri et al., 2014).

A segunda fase do tratamento é feita com os antituberculínicos como isoniazide e rifampicine. Esta parte perdura de acordo com o estado clínico do paciente. Os remédios podem ter efeitos colaterais como febre alta e dores abdominais. Ao sentir tais efeitos deve ser feita a identificação de qual medicamento os está provocando e parar o tratamento com ele imediatamente (Dhahri et al., 2014).

A prevenção da tuberculose ocorre desde a infância, com a aplicação da vacina BCG (Bacilo de Calmette-Guérin) nas crianças ao completar o primeiro ano de vida. Esta vacina tem confiabilidade de $60 \%$ a $90 \%$ e ela parece contribuir para que diminua a mortalidade e o agravamento da transmissão da tuberculose (Abouda et al., 2014).

Segundo pesquisa feita no ano de 2015 pelo Sistema de Informação de Agravos de Notificação (SINAN/MS), o número de casos novos de tuberculose no Brasil até setembro de 2014 foi igual a 50.781. Em 2015 a região norte do país apresentou aproximadamente 6.376 casos; a região sudeste teve aproximadamente 29.024 casos; a região nordeste aproximadamente 16.294 casos confirmados. Segundo o 
Ministério da Saúde, a região sul teve 2.211 casos e a região centro-oeste apresentou 2.256 casos de tuberculose (Barcelar et al., 2014; Chaves et al., 2017).

\section{OBJETIVOS}

Mostrar o número de casos confirmados de tuberculose no Brasil entre 2007 e 2016.

\section{MÉTODO}

Pesquisa realizada no banco de dados DATASUS (http://datasus.saude.gov.br/). Acessando a aba "Acesso à Informação", foi selecionada a opção "Informações de Saúde (TABNET)" e foi selecionada a opção "Epidemiologicas e Morbidade". Após o direcionamento à nova página, clicou-se em "Casos de Tuberculose - Desde 2011 (SINAN)". Selecionou-se a alternativa "Tuberculose - desde 2011" e, em "Abrangência Geográfica", foi selecionada a opção "Brasil por região, UF e município". Em seguida, na nova janela aberta, foi selecionado "Casos confirmados", em Conteúdo, e em Coluna foi selecionada a opção "Não ativa" para todos os tipos de Linha posteriormente selecionadas. Para cada opção foram coletados os dados de todos os anos entre o período de 2009 a 2016 na área "Períodos disponíveis". As Linhas utilizadas foram "Ano de diagnóstico", "Sexo", "Forma", "Tipo de entrada", "Faixa etária" e "Região de notificação". Esta pesquisa foi feita dentro do aplicativo Excel, componente do pacote Office da Microsoft Corporation. Pesquisa bibliográfica foi realizada em livros e artigos científicos, na biblioteca do Instituto Federal de Educação, Ciência e Tecnologia do Amapá, Campus Macapá, situado na: Rodovia BR 210 KM 3, s/n - Bairro Brasil Novo. CEP: 68.909-398, Macapá, Amapá, Brasil.

\section{RESULTADOS}

A Figura 1 mostra o número de casos confirmados de tuberculose no Brasil entre os anos de 2007 e 2016. Os dados mostram que o número de casos se manteve estável por nove anos, apresentando uma queda acentuada no último ano pesquisado. 
Figura 1 Número de casos confirmados de tuberculose no Brasil entre os anos de 2007 e 2016.

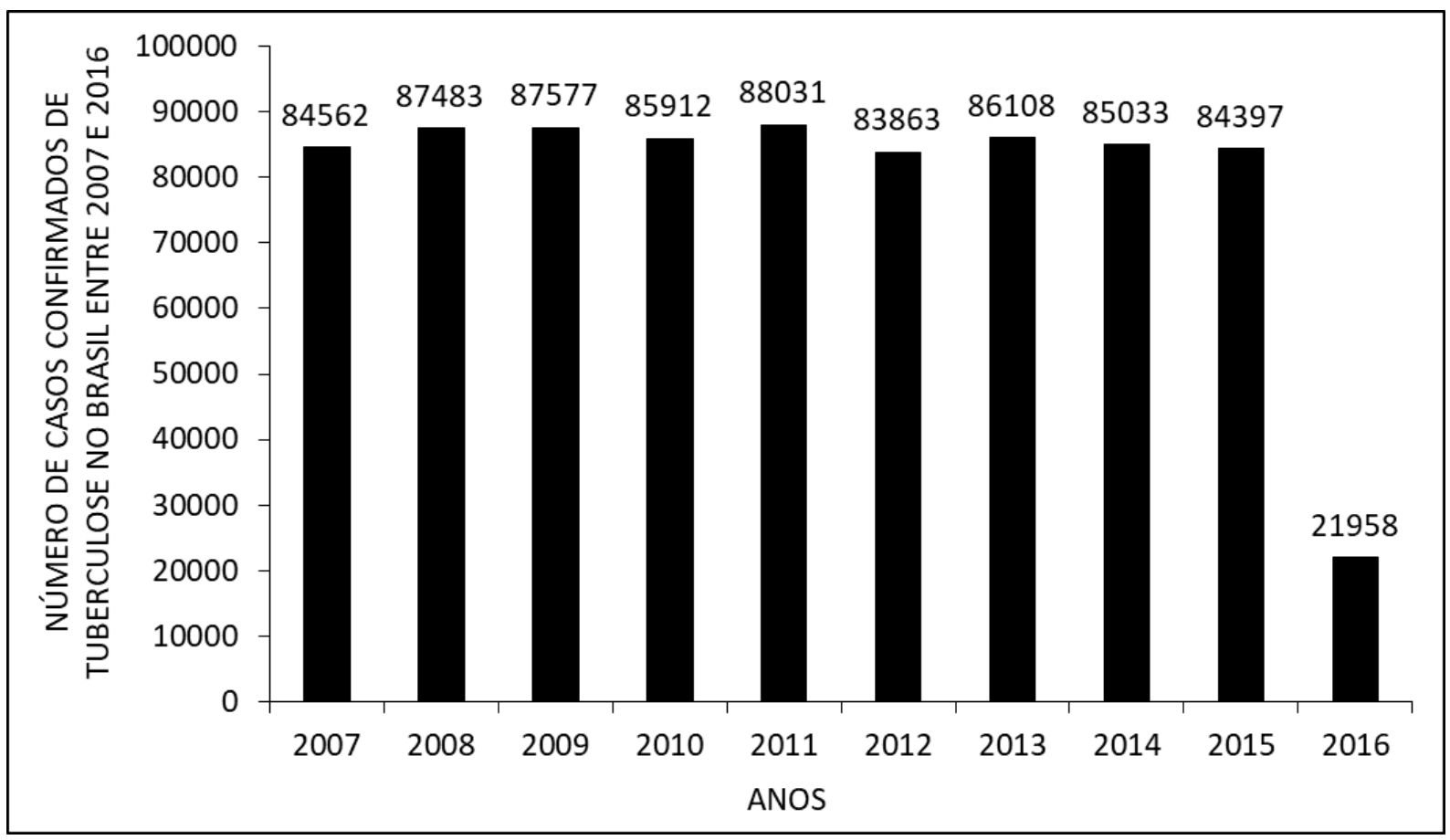

A Figura 2 mostra o número de casos confirmados de tuberculose entre os anos de 2007 e 2016 por regiões do Brasil. Nota-se que a região sudeste possui o maior número de casos confirmados, seguido do nordeste do país, sul, norte e centro-oeste, respectivamente. 
Figura 2 Número de casos confirmados de Tuberculose entre os anos de 2007 e 2016 por regiões do Brasil.

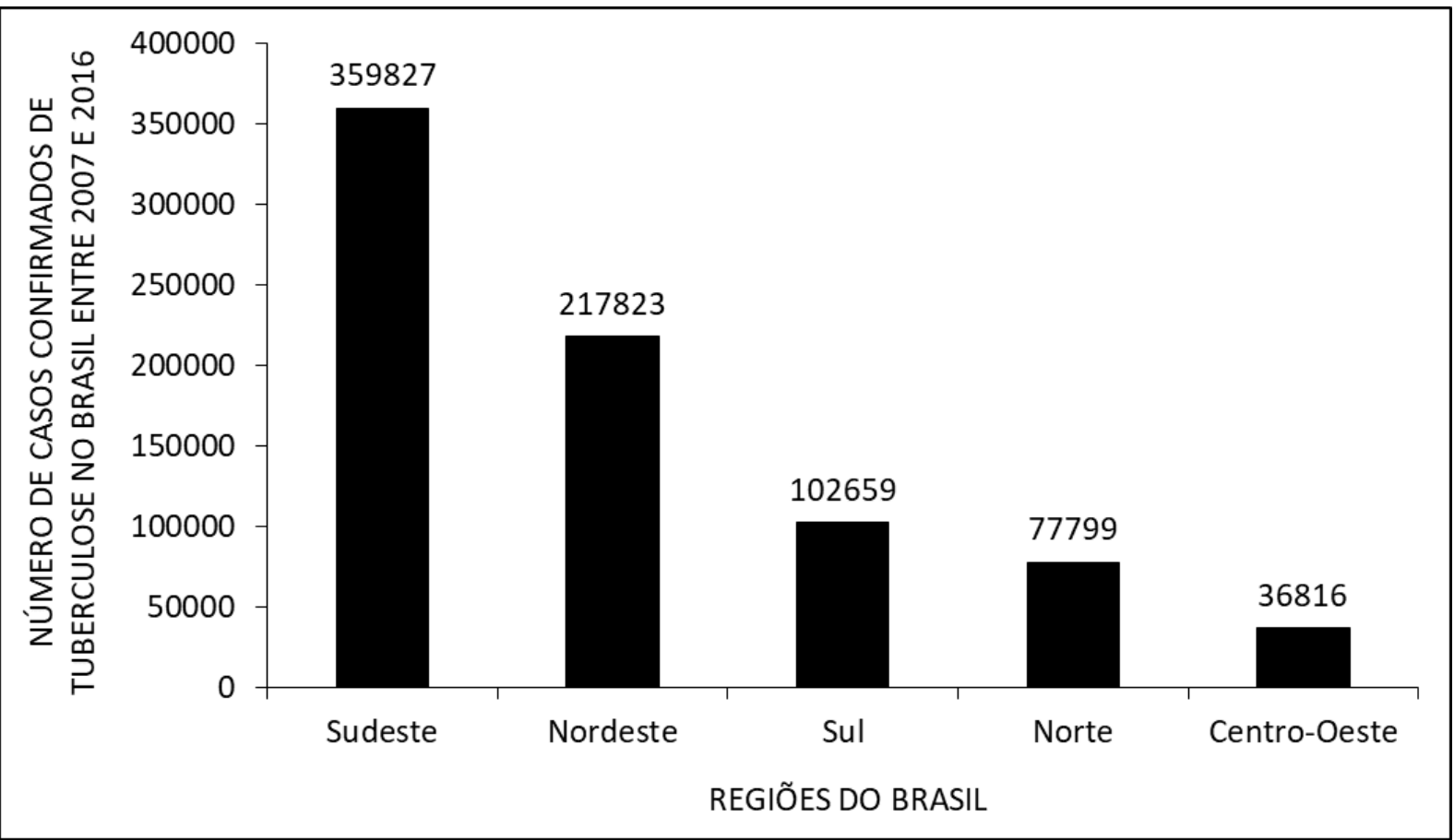

A Figura 3 mostra o número de casos confirmados de tuberculose por faixa etária no Brasil entre 2007 e 2016. Os dados mostram que na faixa etária entre 20 e 59 anos ocorrem o maior número de casos. 
Figura 3 Número de casos confirmados de tuberculose por faixa etária no Brasil entre 2007 e 2016.

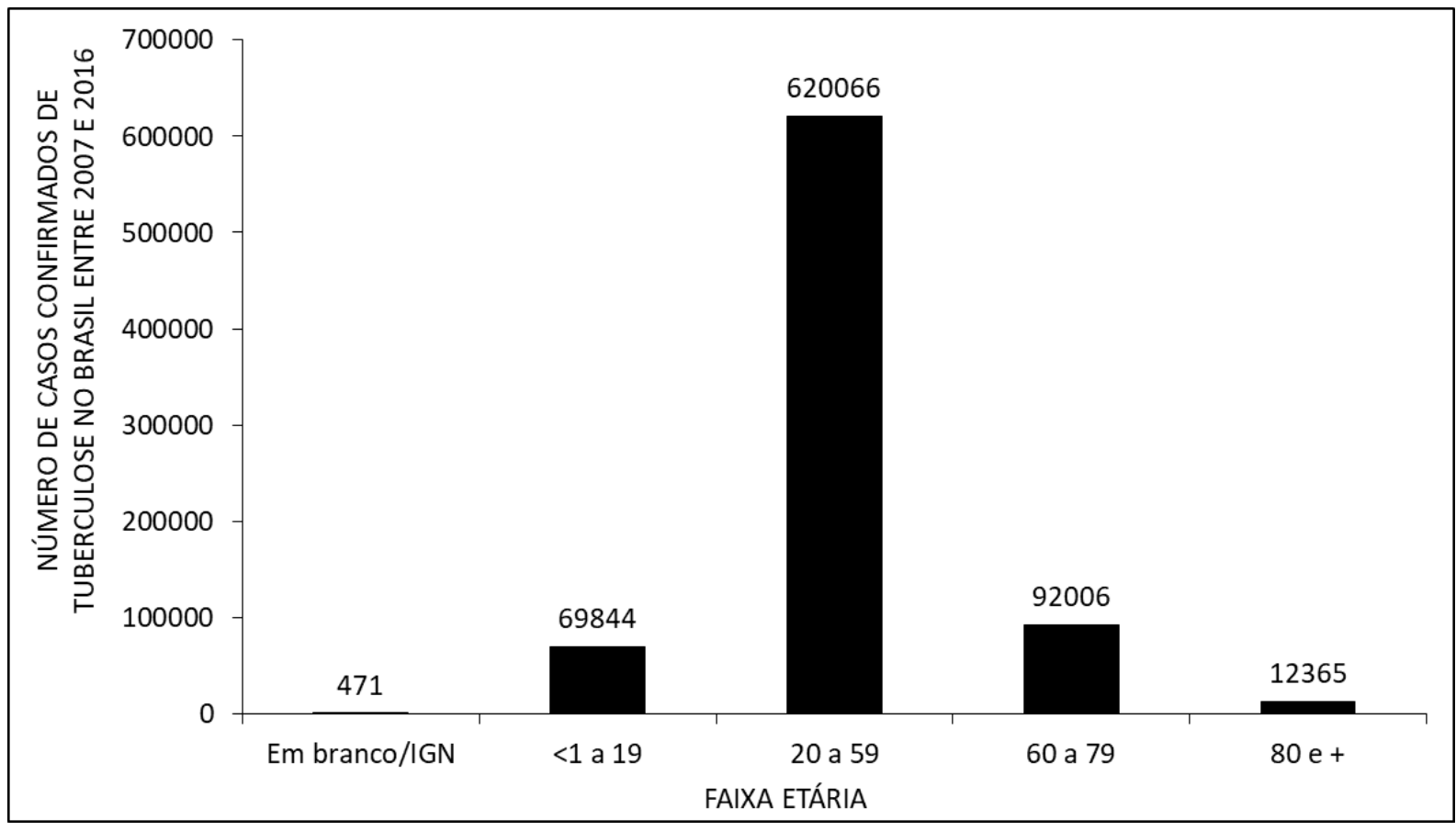

A Figura 4 mostra Número de casos confirmados de tuberculose no Brasil por sexo entre 2007 e 2016. A pesquisa apresenta dados que indicam que o gênero masculino é aproximadamente duas vezes mais acometido de tuberculose que o feminino. 
Figura 4 Número de casos confirmados de tuberculose no Brasil por sexo entre 2007 e 2016.

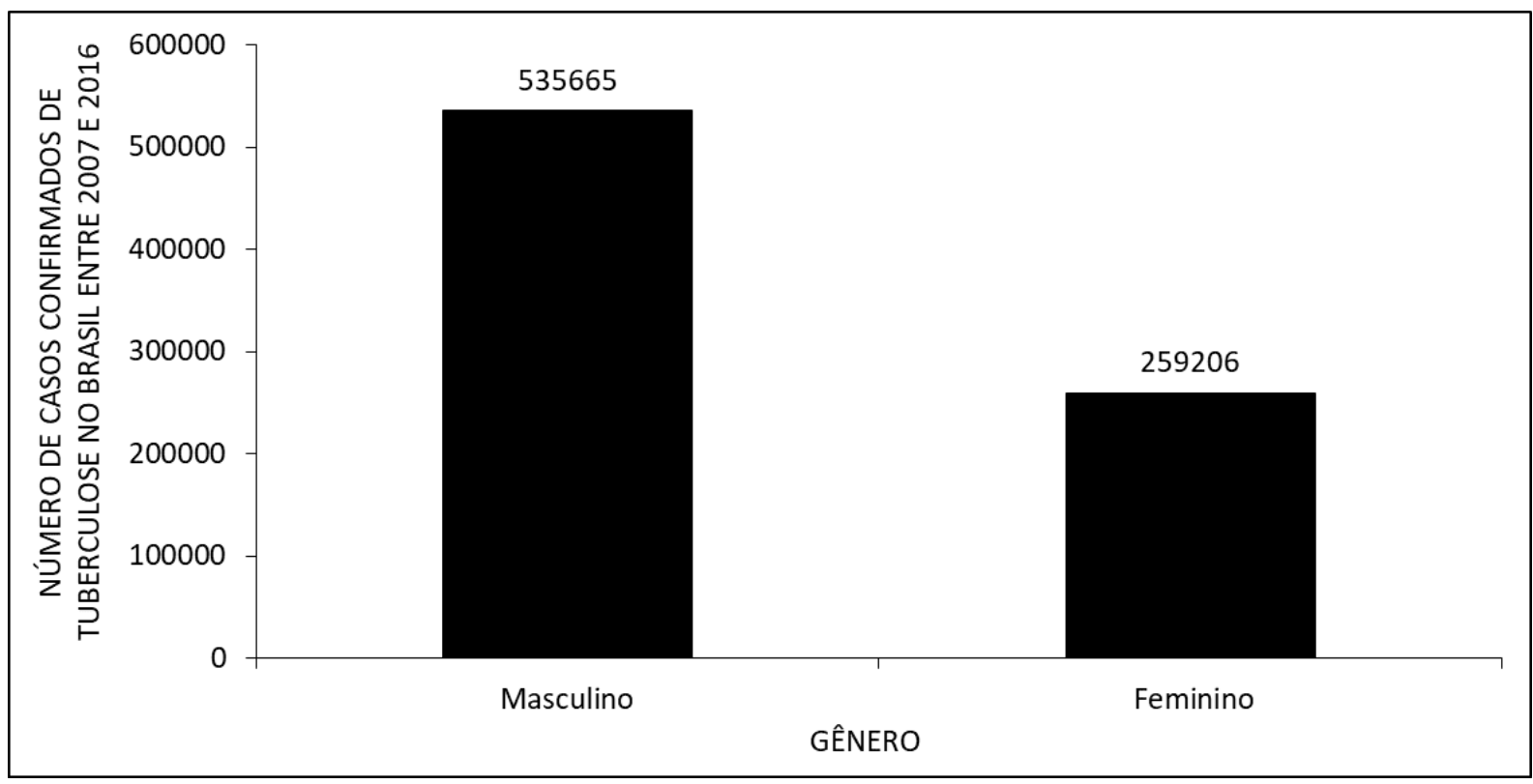

A Figura 5 apresenta o Número de casos confirmados de tuberculose no Brasil entre 2007 e 2016 pela forma da doença. Os casos confirmados com tuberculose pulmonar são muito superiores que os outros dois tipos. Os casos de tuberculose extrapulmonar aparecem em número muito inferior juntamente do número de ambas as formas ao mesmo tempo. 
Figura 5 Número de casos confirmados de tuberculose no Brasil entre 2007 e 2016 pela forma da doença.

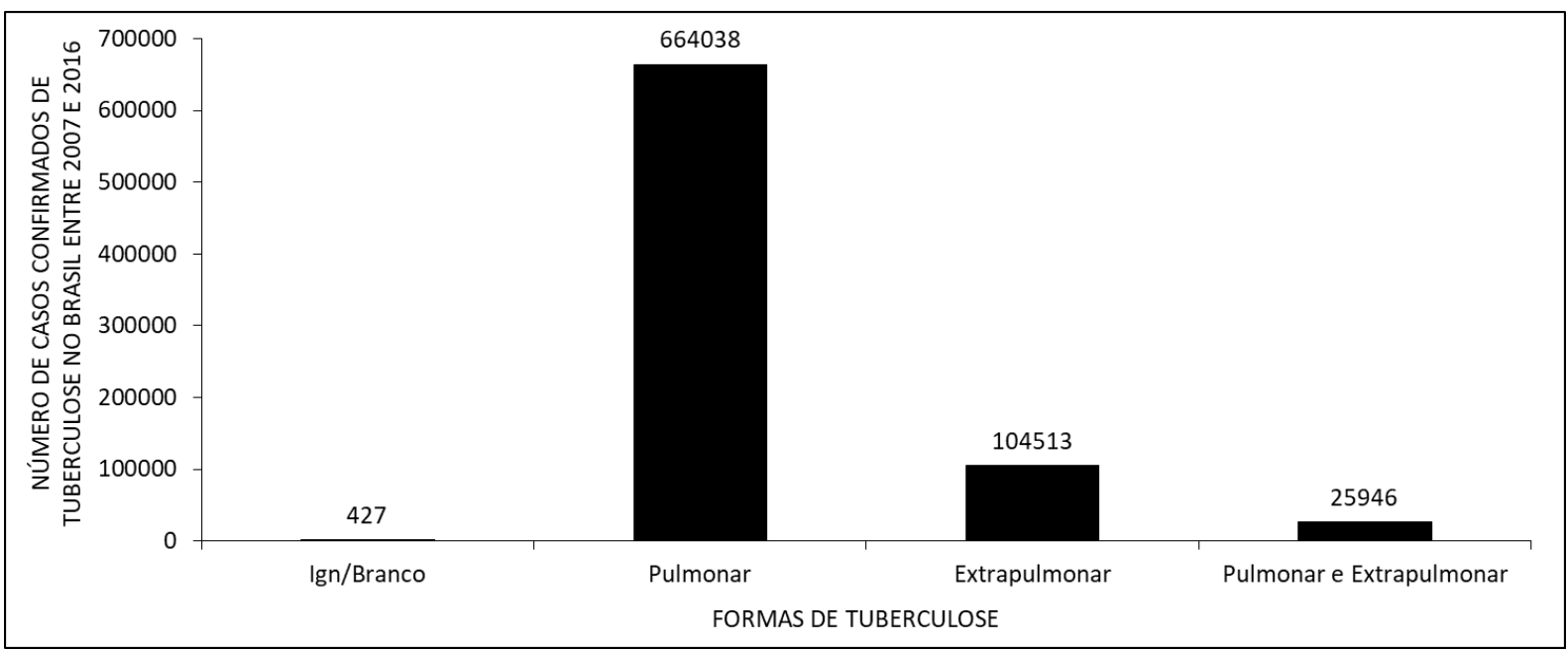

A Figura 6 mostra o número de casos confirmados de tuberculose no Brasil entre os anos de 2007 a 2016 por tipo de entrada. O gráfico mostra que os casos novos possuem um número superior à quantidade de casos dos outros tipos de entrada.

Figura 6 Número de casos confirmados de tuberculose no Brasil entre os anos de 2007 a 2016 por tipo de entrada.

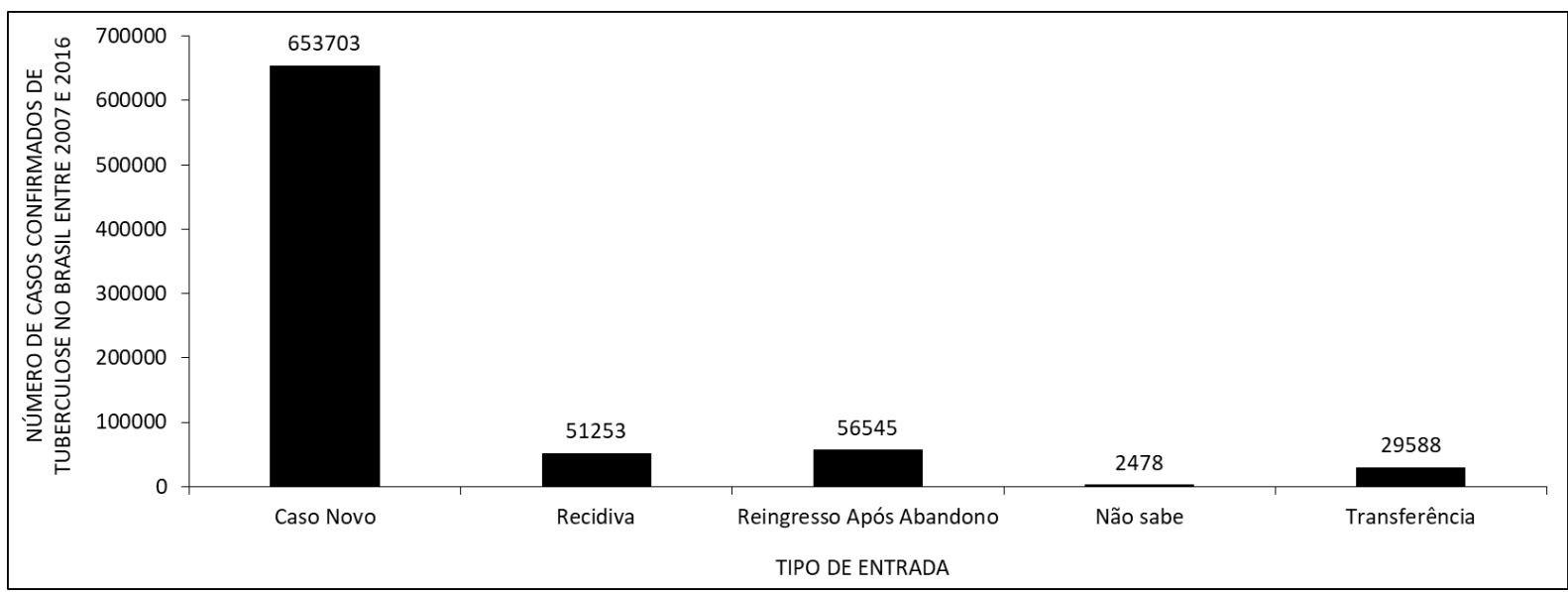

RC: 48850

Disponível em: https://www.nucleodoconhecimento.com.br/saude/casos-confirmados-detuberculose 


\section{DISCUSSÃO}

O índice de casos novos de tuberculose teve um declínio considerável no ano de 2016 (Figura 1). Este fato pode estar relacionado com a melhoria na organização da saúde do Brasil e o melhor preparo dos profissionais da área da saúde no atendimento da tuberculose. O método de diagnóstico feito a partir da avaliação do escarro do paciente também parece influenciar neste declínio, pois ele é a metodologia mais rápida na identificação da bactéria (Cozer et al., 2016; Slim-Saidi., 2015).

Os resultados apontaram o sudeste como a região brasileira com maior incidência da tuberculose (figura 2). Isto pode ter ocorrido por fatores geográficos populacionais. Como a tuberculose é uma doença transmitida pela proximidade, a aglomeração de pessoas facilitaria a sua propagação. Os dados do último censo do Instituto Brasileiro de Geografia e Estatística (IBGE) mostram que o sudeste possui a maior população entre as regiões brasileiras, seguido do nordeste, sul, norte e centro-oeste. Esta é a mesma ordem de incidência nas regiões do país encontradas nesta pesquisa (Abouda et al., 2014).

A Figura 3 mostra que o número de casos novos é maior nas pessoas que têm entre 20 e 59 anos de idade. A duração de proteção da vacina BCG (bacilo de CalmetteGuérin) seria de mais ou menos 15 anos. Como a vacina BCG é aplicada em crianças, as pessoas adultas parecem ficar mais suscetíveis à tuberculose, pois não existe campanha de vacinação para esta faixa etária. Segundo o IBGE (2012), a expectativa de vida do brasileiro é de 74,6 anos, portanto o grupo de indivíduos acima de 80 anos apresenta menor número de casos, possivelmente por estarem em menor número na população (Abiteboul et al., 2010; IBGE, 2012).

A pesquisa mostrou que o gênero masculino é mais acometido pela doença. A falta de cuidado com a saúde que os homens possuem provavelmente explica este dado. Diferente das mulheres, eles não se ocupam da atenção primária à saúde normalmente controlada por meio de visitas de rotina a unidades de saúde. A maioria 
dos poucos que procuram cuidados, em sua maioria, objetiva estética e não saúde, concepção esta gerada por aspectos culturais (Gomes et al., 2011).

O número de casos da tuberculose pulmonar foi maior que a quantidade de casos do tipo extrapulmonar (figura 5). A literatura concorda com estes resultados, pois a TB parece ser transmitida pelo ar de forma rápida em ambientes com muitas pessoas. $O$ que pode explicar o menor número de casos da TBE é a sua forma de propagação. A bactéria precisa entrar no corpo a partir da inspiração e se locomover para outros órgãos diferentes dos pulmões. Isto demanda um tempo maior e estimula uma maior resposta imune (Cozer et al., 2016; Dhahri et al., 2014).

O tipo de entrada com maior incidência da doença foram os casos novos (figura 6). $O$ possível motivo para isso seriam as altas chances de cura utilizando o tratamento correto. Isto parece ocorrer com a maioria das pessoas infectadas pelo Mycobacterium tuberculosis (Daronco et al., 2012; Orofino et al., 2012).

\section{CONCLUSÃO}

A aglomeração de pessoas facilita a propagação da bactéria Mycobacterium tuberculosis. O número de casos da tuberculose pulmonar foi maior que a quantidade de casos do tipo extrapulmonar porque ela é transmitida pelo ar de forma rápida em ambientes com muitas pessoas. Indivíduos adultos parecem ficar mais suscetíveis à tuberculose, pois não existe campanha de vacinação para esta faixa etária. Entretanto as chances de cura são altas quando se utiliza o tratamento correto. Parece ter ocorrido uma melhoria na organização da saúde do Brasil e um melhor preparo dos profissionais da área da saúde no atendimento da tuberculose.

\section{REFERÊNCIAS}

ABITEBOUL, D.; FLORET, D.; AUTRAN, B.; BEYTOUT, J.; COLOMBAN, B.; DUFOUR, V.; LÉVY-BRUHL, D.; JACQUET, A.; JARLIER, V.; MALADRY, P.; NICAND, E.; PATY, M.-C.; POIRIER, C. Pertinence du maintien de l'oligation de 
vaccination par le BCG des professionnels listés aux articles L3112-1, R.3112-1 et R.3112-2 du code de la santé publique. Haut Conseil de Santé Publique, 2010.

ABOUDA, M.; YANGUI, F.; TRIKI, M.; KAMMOUN, H.; KHOUANI, H.; CHARFI, M.R. Prévention de la tuberculose. Elsevier Masson SAS, v. 71, p. 159-167, 2014.

BARCELAR, A.; FRAGA, A. C. P.; Lobo, A. P.; PELISSARI, D. M.; OLIVEIRA, P. B. Panorama da tuberculose no Brasil - Indicadores epidemiológicos e operacionais. Ministério da Saúde, v. 01, p. 57-71, 2014.

BEN AMAR, J.; Dhahri, B.; AOUINA, H.; AZZABI, S.; BACCAR, M. A.; EL GHARBI, L.; BOUACHA, H. Traitement de la tuberculose. Elsevier Masson SAS, v.71, p.122-129, 2014.

CHAVES, J.; TOMILIN, B. A.; BRUN, D.; Fuhr, L. G.; OURIQUE, F.; PILLETTI, K.; KRUMMENAUER, M. L.; PEIXOTO, S. L.; FERNANDES, R.; FREITAS, A. L.; PORCIÚNCULA, A. S.; RODRIGUES, M. T. Perfil dos pacientes com tuberculose que foram atendidos em uma unidade de referência do município de Santa Cruz do Sul RS no período de 2009 a 2013. J. Health Sci., v. 05, p. 31-36, 2017.

COZER, A. M.; ASSIS, L. P. F.; GRACIANO, A. R.; AMÂNCIO, V.; DIAS, D. C. S. Panorama da tuberculose no Brasil. Rev. Educ. Saúde, v. 04, p. 43-50, 2016.

DARONCO, A.; SONDA, E. C.; SILVEIRA, C. S.; BEE, G. R.; PASSOS, P.; BORGES, T. S.; POSSUELO, L. G.; CARNEIRO, M. Aspectos relevantes sobre tuberculose para profissionais de saúde. Rev Epidemiol Control Infect, v. 02, p. 61-65, 2012.

GOMES, R.; MOREIRA, M. C. N.; NASCIMENTO, E. F.; REBELLO, L. E. F. S.; COUTO, M. T.; SCHRAIBER, L. B. Os homesn não vêm! Ausência e/ou invisibilidade masculina na atenção primária. Ciência e Saúde Coletiva, v. 16, p. 983-992, 2011.

IBGE. Expectativa de vida. Disponível em: <https://teen.ibge.gov.br/noticiasteen/7827-expectativa-de-vida>. Acessado no dia 19 de setembro de 2017. 
IBGE; DOU. População Estimada. Disponível em: $<\mathrm{ftp}$ ://ftp.ibge.gov.br/Estimativas_de_Populacao/Estimativas_2014/estimativa_dou_2 014.pd>. Acessado no dia 12 de setembro de 2017.

MJID, M.; CHERIF, J.; BEN SALAH, N.; TOUJANI, S.; OUAHCHI, Y.; ZAKHAMA, H.; LOUZIR, B.; MEHIRI-BEN RHOUMA, N.; BEJI, M. Épidémiologie de la Tuberculose. Elsevier Masson SAS, v. 71, p. 67-72, 2015.

OROFINO, R. L.; BRASIL, P. M. A.; TRAJMAN, A.; SCHMALTZ, C. A. S.; DALCOLMO, M.; ROLLA, V. C. Preditores dos desfechos do tratamento da tuberculose. J Bras Pneumol, v. 38, p. 88-97, 2012.

SLIM-SAIDI, L.; MEHIRI-ZEGHAL, E.; GHARIANI, A.; TRITAR, F. Nouvelles méthodes de diagnostic de la tuberculose. Elsevier Masson SAS, v. 71, p. 110-121, 2015.

Enviado: Abril, 2020.

Aprovado: Abril, 2020. 